\title{
Reirradiation of head and neck cancer in the era of intensity-modulated radiotherapy: patient selection, practical aspects, and current evidence
}

\author{
Yeon Sil Kim, MD \\ Department of Radiation Oncology, Seoul St. Mary's Hospital, College of Medicine, The Catholic University of Korea, Seoul, Korea
}

Locoregional failure is the most frequent pattern of failure in locally advanced head and neck cancer patients and it leads to death in most of the patients. Second primary tumors occurring in the other head and neck region reach up to almost 40\% of long-term survivors. Recommended and preferred retreatment option in operable patients is salvage surgical resection, reporting a 5-year overall survival of up to $40 \%$. However, because of tumor location, extent, and underlying comorbidities, salvage surgery is often limited and compromised by incomplete resection. Reirradiation with or without combined chemotherapy is an appropriate option for unresectable recurrence. Reirradiation is carefully considered with a case-by-case basis. Reirradiation protocol enrollment is highly encouraged prior to committing patient to an aggressive therapy. Radiation doses greater than $60 \mathrm{~Gy}$ are usually recommended for successful salvage. Despite recent technical improvement in intensity-modulated radiotherapy (IMRT), the use of concurrent chemotherapy, and the emergence of molecularly targeted agents, careful patient selection remain as the most paramount factor in reirradiation. Tumors that recur or persist despite aggressive prior chemoradiation therapy imply the presence of chemoradio-resistant clonogens. Treatment protocols that combine novel targeted radiosensitizing agents with conformal high precision radiation are required to overcome the resistance while minimizing toxicity. Recent large number of data showed that IMRT may provide better locoregional control with acceptable acute or chronic morbidities. However, additional prospective studies are required before a definitive conclusion can be drawn on safety and effectiveness of IMRT.

Keywords: Head and Neck neoplasms, Reirradiation, Intensity-modulation radiation therapy (IMRT), Toxicities

\section{Introduction}

Despite intensified treatments for head and neck squamous cell carcinoma (HNSCC), patients still have a high risk of recurrence or second primary tumors occurring within or in close proximity to previously irradiated volume. Long-term follow-up data showed that locoregional recurrences can develop in $16 \%$ to $25 \%$ of patients treated with postoperative chemoradiation for high-risk HNSCC [1] and in 17\% to $52 \%$ of patients treated with definitive chemoradiation for unresectable HNSCC [2]. About 15\% of cases will develop a second primary cancer (2nd primary) in head and neck region within 5 years after initial HNSCC diagnosis. The rate increases to $40 \%$ for long-term survivors [3]. Surgery as salvage therapy

Received 14 March 2017, Revised 27 March 2017, Accepted 28 March 2017.

Correspondence: Yeon Sil Kim, MD, Department of Radiation Oncology, Seoul St. Mary's Hospital, College of Medicine, The Catholic University of Korea, 222 Banpo-daero, Seocho-gu, Seoul 06591, Korea. Tel: +82-2-2258-6259, Fax: +82-2-2258-1532, E-mail: yeonkim7@catholic.ac.kr

(c) This is an Open Access article distributed under the terms of the Creative Commons Attribution Non-Commercial License (http://creativecommons.org/ licenses/by-nc/4.0/) which permits unrestricted non-commercial use, distribution, and reproduction in any medium, provided the original work is properly cited.

www.e-roj.org 
for locoregional recurrence provides the greatest probability of cure, with 5-year survival rate approaching 40\% [4]. Unfortunately, the vast majority of locoregional recurrent cases are unresectable. Although chemotherapy has been the standard of care for patients who are not candidates for surgery, response rates are limited with a median survival time of 7.4 months [5].

Reirradiation is a potentially curative treatment option for some patients with unresectable disease. However, increased risk of severe or life-threatening treatment-related toxicity and tumor radioresistance pose challenges to reirradiation. Because locoregional progression is the most common cause of death in patients with head and neck cancer, obtaining local control may effect on the survival in patients with locoregionally failed disease. In addition, local tumor progression can affect morbidity due to disfigurement in appearance, uncontrollable pain, cancer bleeding, infection, and impairment of speech and swallowing, thus finally resulting in poor quality of life (0OL).

Patients with recurrent or 2 nd primary HNSCC having history of previous radiation therapy are challenging group with heterogeneity. Published data include diverse recurrent or 2 nd primary tumor in the extent and location, prior radiotherapy (RT) parameters, elapsed time since prior treatment, and extent and severity of normal tissue sequelae. Data in current literature are not available on acute and late normal tissue recovery from prior treatment. Lack of data to reirradiation tolerance poses significant challenges or even fear to meet these patients in daily clinic. Recently, high precision RT including intensity-modulated radiotherapy (IMRT) has demonstrated the ability to reduce toxicity and improve disease control. Novel systemic agents and radiotherapy techniques including stereotactic ablative RT (SABR) and proton therapy are also being actively explored. In this study, we sought to formulate widely-applicable scheme based on previous data of reirradiation patients with high precision radiation therapy.

\section{Radiobiologic Aspects of Reirradiation in Head and Neck Cancer}

\section{Radiation resistance}

In vitro analysis of cultured tumor cells from recurrent HNSCC after a curative course of radiotherapy has demonstrated the development of radioresistance [6]. Local control rate was about 30\% following reirradiation for recurrent tumors, which was lower than the expected local control rate of $60 \%$ for initially irradiated head and neck cancer and new primary cancer in previously irradiated areas. Such finding indicates that radioresistance is acquired through high-dose radiation in recurrent head and neck cancer [7].

Whether radioresistance acquired through previous radiation renders subsequent treatments less effective is an important factor when considering retreatment. Radioresistance may partially explain the high prevalence of local recurrences (37\%-60\%) after delivering tumoricidal doses (at least $66 \mathrm{~Gy}$ ) to re-irradiated patients. This suggests that biologically effective and high doses are needed to control gross disease. Recurrent tumors can also be morphologically distinct from de novo tumors, presenting multicentric tumor foci with more dissociated tumor cells in the vicinity [8] Traditional fractionated radiation alone is insufficient for recurrent tumors. Thus, revisiting the use of novel molecular agents or alternative dose fractionation schedules such as hypofractionation or ablative RT with reduced treatment volumes may be warranted to improve tumor control in addressing radiobiologic differences in recurrent HNSCC.

\section{Normal tissue tolerance to reirradiation}

Given inadequate survival of patients with recurrent HNSCC, there is lack of data to see as much as possible late normal tissue complications after 2 nd course of radiation. Currently not enough data are available for normal tissue radiation tolerance especially for partial volume irradiation. Carotid rupture is an uncommon but usually fatal complication. It may occur in approximately 3\% of patients within several months to one year after receiving reirradiation [9]. Spinal cord myelopathy is particularly fearful for radiation oncologist in the clinics as the spinal cord have already been irradiated to above the tolerance dose. However, animal experiments in rhesus monkeys have indicated considerable repair of the spinal cord from initial radiation (repair rate of approximately $76 \%, 85 \%$, and $101 \%$ after 1,2 , and 3 years of initial radiation, respectively) [10]. A recent summary of current literature on this issue has shown an enough evidence that partial repair of subclinical damage in the spinal cord induced by conventional fractionated RT is certain at about 6 months following RT. Such repair rate is increased over till the next 2 or 3 years [11]. For partial cord dose using SABR, a maximum cord dose of $13 \mathrm{~Gy}$ with 1 fraction or $20 \mathrm{~Gy}$ in 3 fractions seems to be at a risk of myelopathy of less than 1\%. Full-dose reirradiation in children with recurrent intracranial ependymoma revealed substantial recovery of spinal cord and brainstem. In summary, current evidence demonstrated that the risk of spinal cord myelopathy is unusual when the elapsed time between radiation is at 
ROJ Radiation Oncology Journal

least 6 months and if cumulative biologically effective dose administered to the spinal cord is remained low 135.5 Gy2 [12]. The reported risk of myelopathy is less than $1 \%$.

\section{Retreatment Strategy for Recurrent or 2nd Primary HNSCC}

\section{Adjuvant radiation therapy following salvage surgical resection}

Locoregional recurrence or 2nd primary HNSCC are mostly heavily treated with surgical resection and RT with or without chemotherapy or both. Salvage surgery has been identified the most effective curative treatment. It is the best choice of treatment for resectable recurrence and with good performance status. According to a meta-analysis of 32 studies, the 5-year survival rate reached up to 39\% after salvage surgery [13]. Reasonably, best chance for cure has been observed in early-stage diseases. Bulky disease with stage T3-T4 recurrence should be considered differently because it is not adequate candidates for salvage surgery [14]. The success of salvage surgery also relates with the location of recurrent tumors. It has a better outcome for recurrent cancer in the larynx and neck node compared with poor sites like as oropharynx and the oral cavity. Although salvage resection is done in selected patients, probability of second recurrence is still high (50\%-65\%).

In 2008, the GORTEC phase III, multicenter, randomized trial [15] compared adjuvant concurrent chemoradiation therapy (CCRT) to just observation after Ro or R1 surgical resection in previously irradiated recurrent HNSCC. Patients were randomized to observation group versus CCRT using hyperfractionation schedule. Twenty-six percent and 29\% of patients had adverse pathologic feature of extracapsular extension (ECE) and positive margins. Adjuvant CCRT improved both locoregional control (LRC, $p<0.0001)$ and diseasefree survival (DFS, $p=0.01$ ), but not overall survival (OS, $p=$ 0.50). Two-year OS reached $45 \%$ in the CCRT group. Acute toxicity increased in $28 \%$ of patients in the CCRT group. Three treatment related deaths were observed during CCRT. At 1 and 2 years, grade $3-4$ toxicity were $26 \%$ and $39 \%$, respectively, in the CCRT group compared to $9 \%$ and $10 \%$, respectively, in the observation group $(p=0.06)$. Treatment mortality was higher ( 5 vs. 0). There were several smaller prospective or retrospective series results. The following conclusions are drawn from these studies [16]. First, only patients with high-risk adverse pathologic features should be considered for postoperative adjuvant reirradiation (positive surgical margins and ECE).
Optimal reirradiation strategy for head and neck cancer

Second, grade 3-4 high chronic toxicities may develop in more than $1 / 3$ of patients. Third, reirradiation related death was observed up to $8 \%$ of patients. Fourth, even with high rate of morbidities, half of patients can survive longer than 2 years. Fifth, adjuvant reirradiation with or without chemotherapy can improve LRC and DFS compared with salvage surgical resection alone, however, it has no prolongation of OS.

It is currently uncertain whether the addition of concurrent chemotherapy to reirradiation can improve treatment outcome. Reirradiation morbidity after salvage resection should be investigated. Reconstruction with vascularized tissue flap can reduce small vascular damage and overlying skin against chronic morbidity. A retrospective study of 12 patients who had introduced microvascular free flap reconstruction for recurrent or 2nd primary HNSCC in previously radiated patients has showed that free flaps reconstruction can make possible successful resection, which may also reduce the incidence of severe late radiation complications such as skin sloughing, soft tissue fistula, and great vessel rupture and also reduced the risk of treatment-related death [17].

\section{Reirradiation for unresectable disease}

Nonsurgical salvage therapies are usually applicable to patients with a bulky disease and to those who are unsuitable for curative resection. Two randomized trials (GORTEC 9803, RTOG 04-21) [18] have compared chemotherapy alone and chemo-irradiation. They were early closed because of poor enrollment. In unresectable recurrent HNSCC, treatment decisions based on results from systematic reviews and expert panel recommendation have shown that RT with or without chemotherapy can improve $\mathrm{OOL}$ and chance of long-term control compared to best supportive care.

The Radiation Therapy Oncology Group (RTOG) has successfully performed two multi-institutional prospective phase II trials. In RTOG 9610 trial [19], 86 patients were recruited and radiotherapy was delivered with $1.5 \mathrm{~Gy} /$ fraction twice per day, concurrently with hydroxyurea/5-fluorouracil (5FU) separated by 1 week of rest for 4 cycles. The late grade $3 / 4$ toxicities were observed in $19.4 \%$ of patients. The OS was $15.2 \%$ at 2 years. There were $6(7.6 \%)$ treatment-related deaths. The following RTOG 9911 study [20] was conducted from 2000 to 2003. It enrolled 105 patients who were treated with the identical radiation scheme (IMRT was included) but with a different concurrent chemotherapy agents (cisplatin/ paclitaxel). The incidence of grade $3 / 4$ late toxicities was high reported up to $33.8 \%$. Eight patients (8\%) of treatment-related deaths occurred. At 2 years, PFS and OS were 15.8\% and 


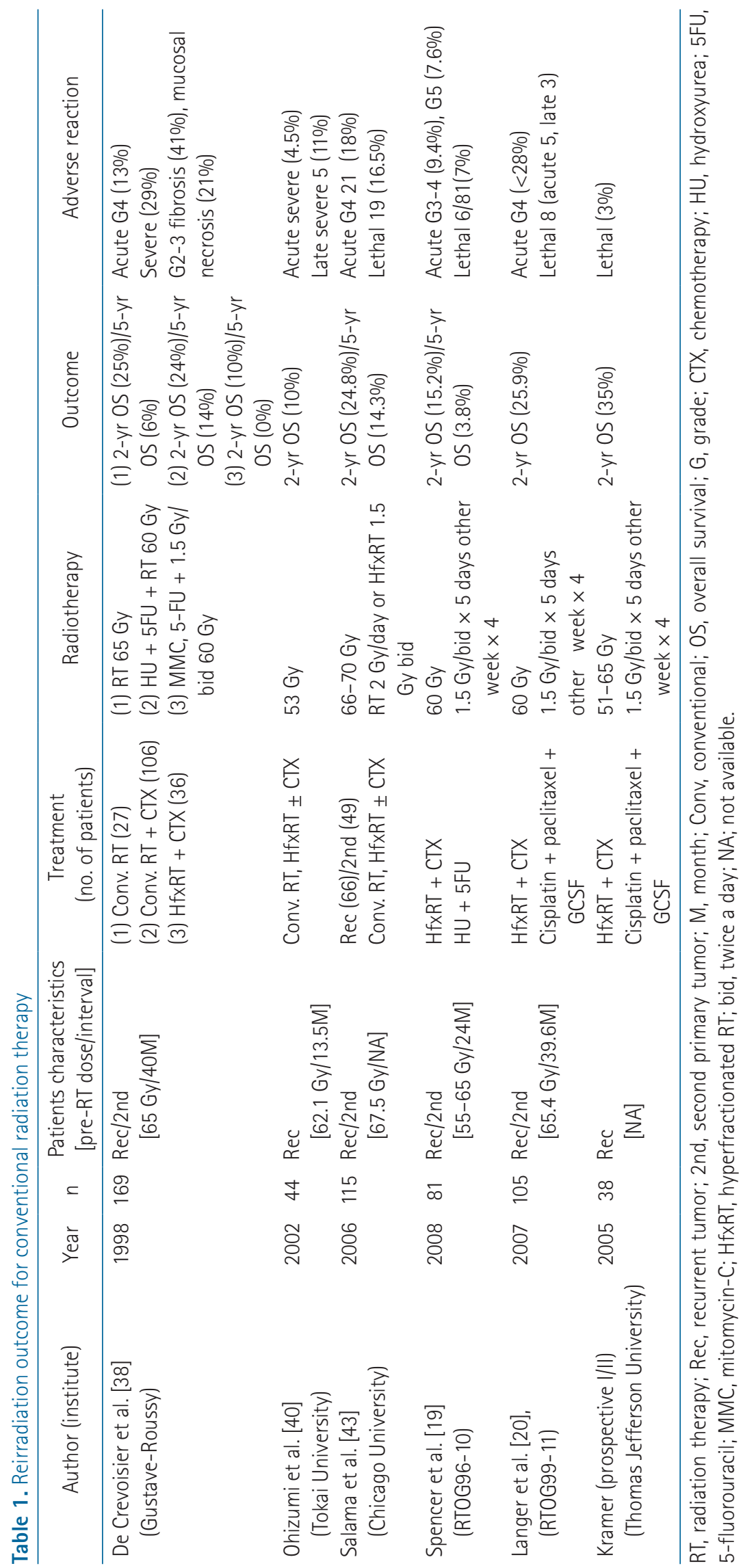


$25.9 \%$, respectively. These were relatively superior treatment outcomes (2-year OS 25.9\% in RTOG 9911 vs. 15.2\% in RTOG 9610; $p=0.0444$ ) compared to the other two RTOG trials. Table 1 summarized studies on reirradiation with or without chemotherapy in recurrent, unresectable HNSCC. Results from those studies represented that $1 / 4$ to $1 / 3$ of patients were locoregionally controlled with 2-year OS rates of $10 \%$ $30 \%$, although long-term survivors after reirradiation were uncommon. Severe toxicities above grade 3/4 could happen in up to $40 \%$ of re-irradiated patients. Almost $10 \%$ of patients might suffer from treatment-related death [16]. Apparently, the outcomes of studies showed considerable difference that primarily depends upon included patients and the intensity of reirradiation. Until recently, there are no randomized studies on defining optimal reirradiation treatment schemes. As shown in Table 1, previous studies had wide differences in patient and tumor characteristics, and treatment-related factors so those studies make it impossible to draw any clinically meaningful conclusions. Therefore, it leaves a lot of questions concerning the most effective reirradiation regimen (split-course vs. continuous-course; once daily vs. hyperfractionation). The therapeutic effect and toxicities of concurrent chemotherapy should be defined.

\section{Palliative chemotherapy alone}

Chemotherapy as a single therapeutic modality only plays as a palliative role. Patients with palliative chemotherapy alone only have a median survival of 7.4 months. A phase III EXTREME trial [5] has demonstrated 3 months survival benefit from 7.4 months to 10.1 months (hazard ratio [HR], 0.80; $p=0.04$ ) by addition of cetuximab to conventional chemotherapeutic agent (platinum $+5 \mathrm{FU}$ ) in recurrent and unresectable HNSCC. However, result of early randomized trials including recurrent/ metastatic HNSCC patients have shown disappointing 5-year survival rate of $3.6 \%$ following platinum-based combination chemotherapy [21].

\section{Radiation Therapy Technique}

Preferred reirradiation technique is delivering radiation using brachytherapy, SABR, or external beam radiotherapy (3-dimensional conformal therapy [3D-CRT], IMRT or proton beam therapy) combined with or without systemic agents (chemotherapeutic or immunologic agents) in presence or absence of preceding debulking surgery.

IMRT with modern image guidance has been commonly attempted in practice. Because of its potential benefit in reduced toxicity and treatment efficacy compared to conventional radiation therapy, many recently published studies on reirradiation have made the use of IMRT or SABR. Those studies are summarized in Tables 2 and 3. As shown in Table 2 or 3 , the numbers of patients are small in each study and also wide heterogeneity has been observed in RT parameter and presence or absence in salvage surgery and systemic therapy. No definitive survival advantage has been observed with IMRT or SBRT compared with conventional radiation therapy till now. However, improvement in local control and advantage of acute/late toxicities are apparent, although it is considered that irradiated volume tends to be smaller when IMRT or SABR are utilized. Certainly, no firm conclusions can be drawn as to toxicity or treatment-related mortality. This might be due to a lack of adequate data in older series. Lee et al. [22] reported retrospective data of 105 patients who were treated with curative intent reirradiation using either conventional RT technique (31 patients) or IMRT (74 patients). IMRT technique has achieved significantly better locoregional relapse free survival over conventional techniques at 2 years (52\% vs. 20\%; $p<0.001$ ). Furthermore, IMRT technique is identified as an independent prognostic factor in multivariate analysis ( $H R$, 0.37). Another study has reported chemoradiation results for recurrent HNSCC [23]. Late G3-4 adverse reactions developed in 7\% of patients treated with IMRT compared to $16 \%$ in conventional RT without treatment related mortality [23].

SABR is a highly conformal and precisely-targeted radiation delivery technique that can focus high dose of radiation to a very small volume area in 1 to 5 fractions. Although there are only limited data with SABR, it may have curative potential particularly for limited small lesions. SBRT also has practical advantages for patients in poor general condition because it does not have hematologic or systemic toxicities. An early retrospective study of SABR for primary, recurrent, or metastatic HNSCC has gained 1-year tumor control rate of $60 \%$ with a median survival of 7 months [24].

In a phase I dose-escalation trial of SABR in reirradiation of HNSCC, total 44 Gy in 5 fractions was delivered without doselimiting acute toxicity [25]. In regarding to adequate radiation dose using SABR, 35-44 Gy in 5 fractions is associated with improved local control compared to those receiving less than 35 Gy without an increase in acute or late toxicity [26]. In contrast, another study has reported high rate of late complication after reirradiation with SABR (median, 30 Gy in 5 fractions) showing carotid blowout rate of 17\% [27]. A retrospective matched cohort study compared SABR (40 


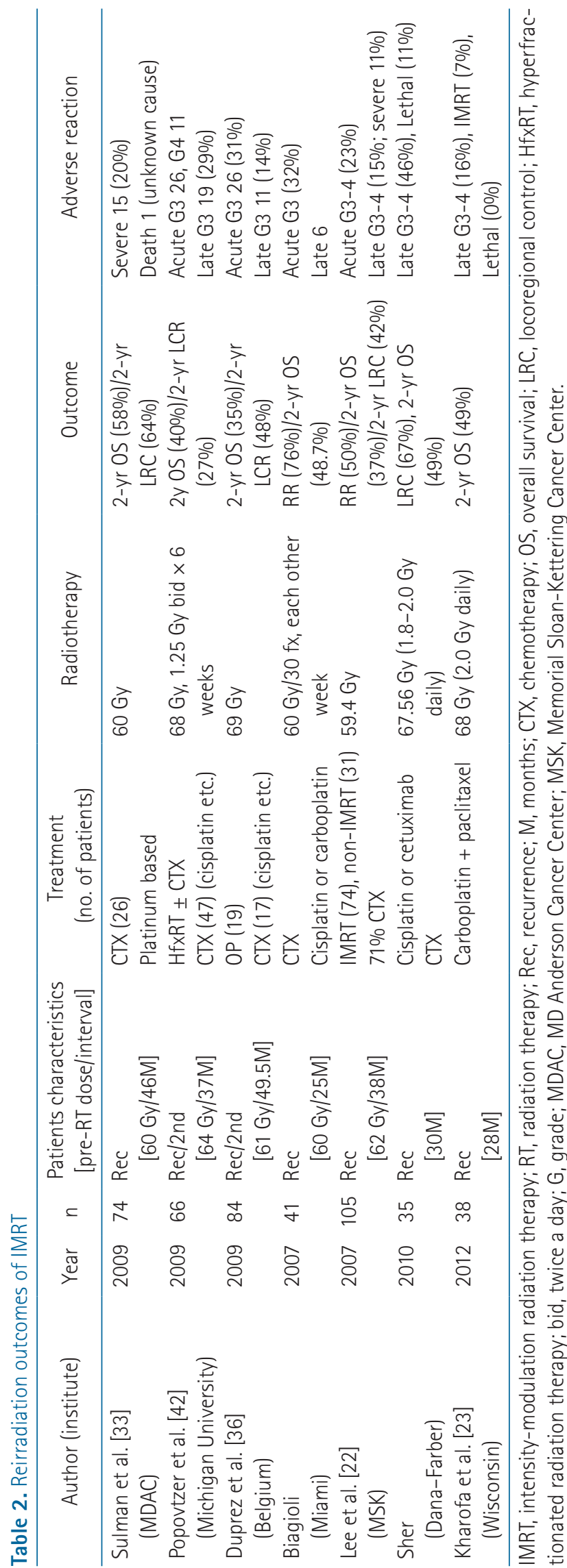

Gy/5 fractions) with or without cetuximab has shown that the additional cetuximab to SABR can increased local control (49.2\% vs. $33.6 \%$ at 2 years) and also OS (53.3\% vs. $21.1 \%$ at 2 years) in comparison to SABR alone [28] without difference of late grade $3 / 4$ toxicities in patients who received concurrent cetuximab.

Proton therapy, a new novel radiation modality with a delicate range of beam but no exit dose, has also been reported in superiority as reirradiation tool such as in nasopharyngeal cancer [29]. In considering significant toxicities to normal tissues with reirradiation, highly precision radiation modalities should be chosen to reduce the volume of reirradiation as small as possible.

\section{Latest Clinical Data from Reirradiation using IMRT}

Recent review on 15 years (1999-2014) of large sized data from MD Anderson Cancer Center using IMRT has clarified the predictors of outcomes and toxicity in re-irradiated patients with HNSCC [30]. Total 206 patients including 173 with HNSCC histology were retreated with curative aim. One hundred four patients (50\%) underwent salvage surgery while 102 patients treated with non-surgical treatment. With a median 24.7 months of follow-up after reirradiation, clinical outcomes were worse for HNSCC patients in 5-year LRC, PFS, and OS rates $(53 \%, 22 \%$, and $32 \%$, respectively), than for non-HNSCC patients $(74 \%, 59 \%$, and 79\%, respectively) [30]. Favorable prognostic factors in multivariate analysis after salvage surgery were neck retreatment and lack of ECE. For non-surgical treatment, better outcomes were noted in nasopharynx subsite and complete responder to induction chemotherapy. However grade 3 toxicities occurred in 32\% of patients at 2 years, $48 \%$ at 5 years, and dysphagia or odynophagia were observed most frequently [30]. Most important factors associated with grade 3 toxicity was reirradiation volume $>50 \mathrm{~cm}^{3}$ and whether or not concurrent chemotherapy was used. Interestingly, there was no grade $3 / 4$ late toxicity in patients whose reirradiation volume was less than $25 \mathrm{~cm}^{3}$ [30]. Recent update on reirradiation results with IMRT with or without salvage surgery acquired promising local control in half of the patients, and also increased long-term survival in selected patients. However, treatment-related toxicity was still high up to 30\%-50\%. Therefore, highly conformal and precise radiation delivery to very limited volume is essential to avoid critical late toxicities. 


\section{Optimal Radiation Volume in Reirradiation of Head and Neck Cancer}

The main challenges in reirradiation of patients are determining the extent of target volume and whether or not to irradiate the uninvolved elective nodal area. At this point, several findings from previous studies should be considered. First, differences in the pattern of metastases (the incidence and geographic distribution of metastases), in other words, unexpected lymph drainage pattern has also been found in patients with previously treated neck [31] with surgery or radiation. Second, high rates of local failures and systemic metastases, both of them are observed after retreatment. This can markedly reduce the potential therapeutic benefit anticipated from elective nodal irradiation. Furthermore high rate of grade $3 / 4$ toxicities were noted after large volume of reirradiation, so the optimal reirradiation volume is not definitely settled now. Two RTOG phase II studies $[19,20]$ on reirradiation with concurrent chemotherapy have set clinical target volume (CTV) with $2 \mathrm{~cm}$ margin surrounding the recurrent gross tumor volume. With an effort to reduce severe toxicity caused by reirradiation, many studies have targeted diseases with restricted volume and avoided elective nodal reirradiation. With PET-CT based treatment planning, marginal local failure can be effectively decreased and $0.5-\mathrm{cm}$ margin around recurrent gross disease is taken popularly [16]. Surgical series and recent IMRT series have found that those who develop a second failure after retreatment experience local recurrence in still high dose volume area (40\%) and distant metastasis (22\%) [30]. Other patterns of failure analysis also suggest that limited reirradiation volumes without elective reirradiation of nodal areas are sufficient [32].

\section{Optimal Dose Fractionation Scheme according to RT Technique}

Until now, objective comparison studies of various reirradiation schemes have not been performed. Data collected from non-recurrent HNSCC patients have suggested that hyperfractionated regimens would be the most effective scheme in sparing late-responding normal tissues. Generally, previous fractionation studies have used hyperfractionation schedule based on the assumption that small fractionation size might decrease late reaction. Moreover, treatment strategies to overcome the reduction of radiation doses, concurrent chemotherapy are preferred.

RTOG $[19,20]$ phase II trials and another study [33] adopted relatively high fractional dose of 1.5 Gy with bid schedule in 1 week-on and 1 week-off alternative fashion. These prolonged hyperfractionation regimens did not demonstrate advantages in treatment efficacy or toxicity compared to continuous course hyperfractionation of 1.2 Gy or conventional fractionation of 1.8-2 Gy/day. Contrarily, these prolonged regimens employed several planned treatment interruption. They are necessary to reduce acute toxicities, but they could be radiobiologically detrimental to local tumor control. Another single institution reports of reirradiation have usually used continuous conventional fractionation with only acute treatment-related deaths of $0 \%-1 \%[22,33]$ compared to $5 \%$ $10 \%$ in studies using prolonged accelerated hyperfractionated weekly cycle regimen used in RTOG $[19,20,33]$.

With rapidly applying precision RT technique from 3D-CRT to IMRT, SABR and proton, the effect of fraction size on reirradiation HNSCC is difficult to estimate. Studies using IMRT usually used standard fractionation without definite increase in late toxicity (Table 2). Several studies of SABR conducting hypofractionated treatment schedules are summarized in Table 3. It remains unclear if delicate focusing to target can reduce potential late normal tissue complications or hypofractionated RT can compensate for less sublethal and potentially lethal damage repair for treatment efficacy. However, hyperfractionated RT will be continuously considered and play a potential role in reirradiation, particularly in patients with recurrent tumor in close proximity to critical organ or those with significant chronic sequel from prior irradiation.

\section{Site Specific Consideration in Reirradiation for Nasopharyngeal Cancer}

An ample of data on reirradiation has been built up for nasopharyngeal carcinoma. Hong Kong Nasopharyngeal Study Group database has evaluated patients of 1st isolated local recurrence and received salvage treatment, including external radiotherapy, brachytherapy, and/or surgery [34].

The 3-year actuarial OS rate for patients with isolated local failure was $74 \%$. As surgery can only be adopted in limited small volume tumors, reirradiation was most often chosen as a treatment. Whatever radiotherapeutic modality was used (intracavitary brachytherapy, IMRT or SABR/SRS), the 3-year local control rate was 75\%-85\% [34]. Therefore, treatment modality should be selected based on each patient's characteristics and tumor factors such as age, performance status, tumor location, size, histology, past treatment, and the willing of the patient. Major complications included 


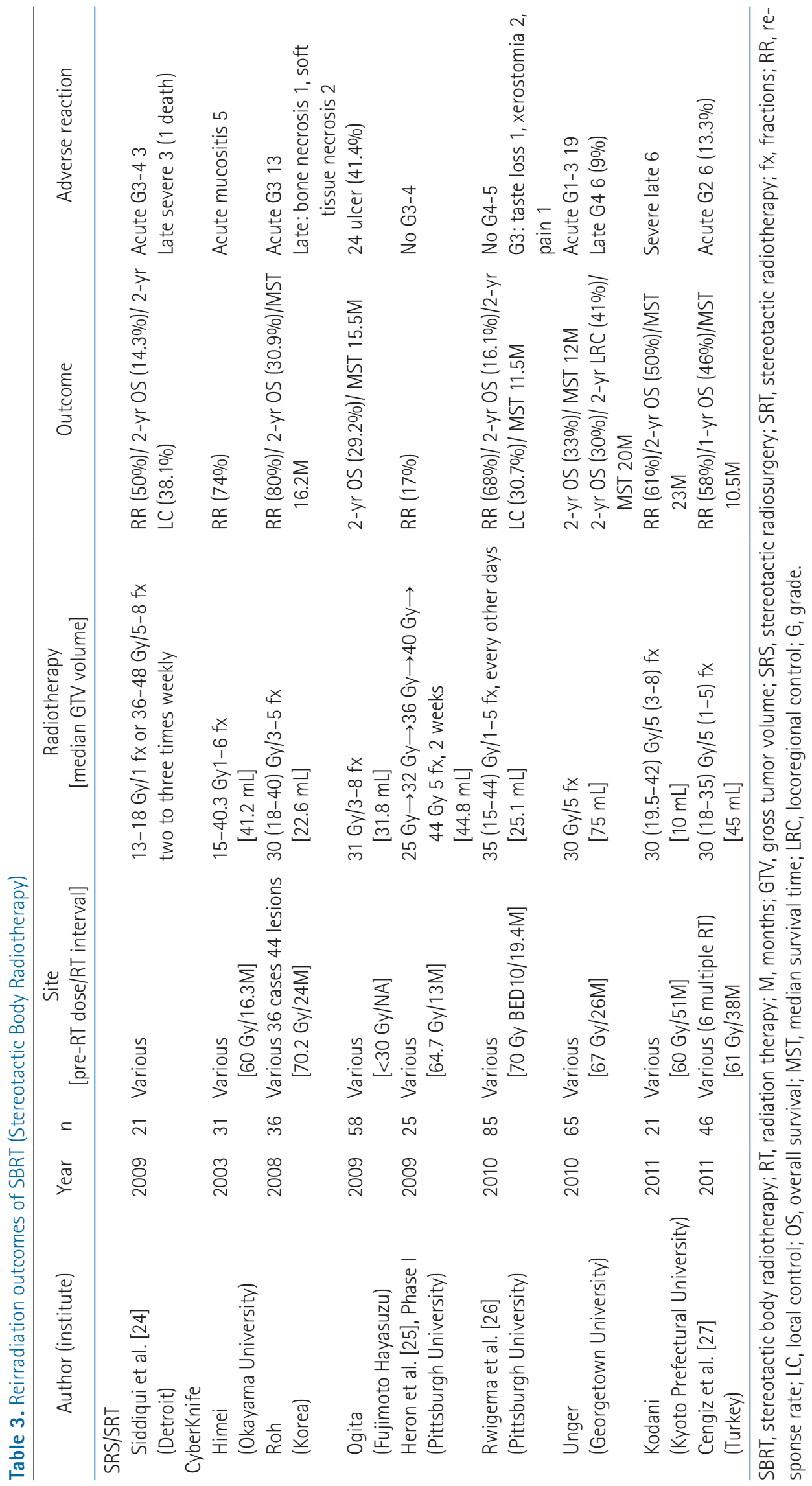


ROJ Radiation Oncology Journal

nasopharyngeal necrosis with headache, necrosis of cervical vertebrae with atlanto-axial instability, temporal lobe necrosis, and cranial nerve palsy [34]. The most morbid complication was temporal lobe necrosis occurring in about $12 \%$ of retreatment patients, with a mortality rate of 65\% [34].

\section{Treatment-Related Toxicity Associated with Previous Irradiation}

Reirradiation alone or CCRT with or without salvage surgery offers a small but actual chance of long-term survival, which has to pay a price of substantial treatment-related morbidity and mortality. However, many radiation oncologists perform high-risk, high-reward therapy if the patients have been left untreated that may lead to grave prognosis. The exact toxicity induced by reirradiation is very difficult to approximate as toxicity might be rooted from one or more of several prior therapies (RT, chemotherapy, surgery, and CCRT). The reported rates of adverse effects in the literatures tend to be underestimated.

\section{Acute toxicities}

Primary CCRT is associated with as much as $71 \%-77 \%$ of grade 3-4 mucositis when compared to reirradiation group (14\%-26\%) [35]. Unexpected low rate of mucositis in reirradiation series is probably due to the smaller irradiation volumes are commonly used. Unfortunately, the rates of treatment-related death in reirradiation patients (5\%-19.9\%) are higher. This may be related to the fact that functional reserve is compromised in heavily pretreated patients rather than direct cause of toxicity. In addition, high radiation doses might have cumulative impact on normal tissues. Most commonly observed causes of death in reirradiation patients are pneumonia due to leucopenia or aspiration and fatal hemorrhage from carotid blowout syndrome.

\section{Late toxicities}

Current data for assumption of risks or dose constraints of soft tissues, bone, and neurovascular structures after reirradiation are insufficient, especially when hypofractionation or SABR is given to patients. Because of poor survival rate and grave prognosis with recurrent HNSCC, many patients may not survive long enough to see potential late normal tissue complications. Swallowing difficulty seems to be the most frequently observed toxicity reported up to $50 \%$ or more in treated patients [30]. The most critical consequences of reirradiation are late neurologic toxicity such as spinal
Optimal reirradiation strategy for head and neck cancer

cord myelopathy, carotid artery rupture (CAR), and osteoradionecrosis (ORN) in the irradiated bone.

\section{1) Spinal cord myelopathy}

Spinal cord myelopathy is a particularly feared late complication to a radiation oncologist as partial volume of the spinal cord have already irradiated 45-50 Gy from the previous radiation treatment. However, animal experiments [10] and sufficient human data have suggested that spinal cord undergo substantial recovery from the initial radiation dose after at least 6-month interval with a low risk of myelopathy despite accumulation doses of more than BED 100 Gy3 [36]. RTOG phase II studies of reirradiation $[19,20]$ and several institutional experiences have restricted the cumulative spinal cord dose to 50 or $60 \mathrm{~Gy}$ [37]. On the other hand, others have delivered somewhat higher cumulative spinal cord doses on the assumption that spinal cord tissue recovery would happen up to 50\% of prior dose following 1 year [33]. All demonstrated a less than $1 \%$ risk of spinal cord myelopathy.

\section{2) Carotid artery rupture}

Carotid artery rupture is an uncommon but usually a fatal complication of reirradiation according to published literature [9]. It may arise in about 3\% of patients at a median of 7.7 months with $76 \%$ of events being fatal [9]. Risk factors for carotid artery rupture include tumor recurrence accompanying chronic infection, surgery (pharyngocutaneous fistula and neck dissection), poor nutrition, and chronic inflammation (long-term tracheostomy and nasogastric tubes). Radiation therapy scheme may impact on risk of carotid blowout. Carotid blowout has been found in low probability when treated with a continuous conventional fractionation or hyperfractionation in comparison with accelerated fractionation regimens (1.3\% vs. $4.5 \% ; p=0.002$ ) [16], although definitive conclusion could not be drawn about the impact of fractionation because of a heterogeneous patient population and treatment parameters.

\section{3) Osteoradionecrosis of bone and mandible}

Mandibular ORN reported predominantly in old data using conventional opposed two bilateral ports up to 10\%-15\% [38]. Not surprisingly, risk of ORN was correlated with dose and volume of irradiated mandible [38]. Because of recent advances in delicate planning and targeting of modern RT techniques (IMRT, SABR), occurrence of ORN reduced significantly, ranging from 0\%-7\% (median, 0\%) [38]. Obviously, options such as reconstructive surgery to remove necrotic tissue and preventive swallowing exercise programs are also important 
as much as precise RT planning and dose delivery for reducing the risk of ORN. Besides the mandibular bone, other tissues such as the laryngeal cartilages and brain are also sensitive to radiation. Consequently, the normal tissue in close proximity to the tumor can influence on the type of radiation-induced toxicity.

\section{Prognostic Factors Associated Reirradiation Outcomes}

According to present literature evidence [39], several prognostic factors associated with reirradiation outcomes are summarized in Table 4.

\section{Debulking surgery}

Surgery has provided additional benefit of removing radioresistant disease that make a great chance of cure. GORTEC multicenter groups have randomized 130 patients to surgery with or without adjuvant radiation with concurrent 5FU/hydroxyurea [16]. Although, no overall survival advantage has been addressed so far for patients with adjuvant radiation, PFS was significantly improved in the adjuvant therapy group [16]. However, acute and late complications were increased in that group.

Table 4. Reported prognostic factors for survival and risk factors for adverse reactions

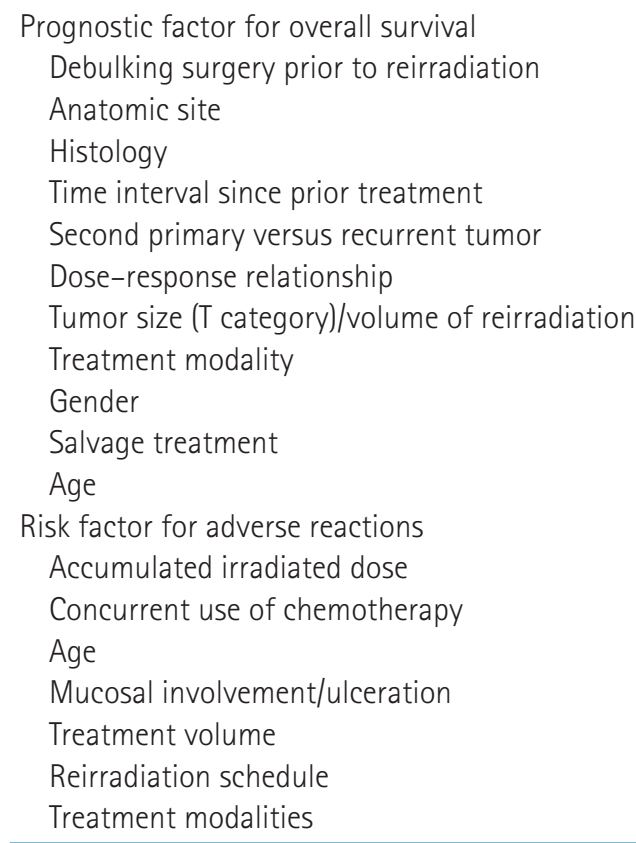

\section{Tumor size (rT1-3 vs. T4) or irradiation volume}

Patients with a non-bulky, small volume of disease prior to reirradiation are most likely to benefit from aggressive locoregional treatment [38], absolutely a higher dosage of RT could be administered to the smaller target than that for a larger tumor [40].

\section{Anatomical site}

Among head and neck sites, good locoregional control have been observed in patients with nasopharyngeal, laryngeal cancer, or lateral neck recurrence [30], whereas oral cavity and hypopharyngeal cancers have relatively poor prognosis [40] after retreatment.

\section{Time interval since prior irradiation}

Many studies have demonstrated that time interval since prior irradiation is an important prognostic factor of reirradiation failure.

In RTOG 9610, the 1-year survival rate for patients reirradiated within 3 years of prior radiotherapy was only $35 \%$. However, it was $48 \%$ for patients re-irradiated more than 3 years later from prior RT [19]. Other study reported reirradiation delivered with 24 months or more interval had a longer median survival time than for patients within 1 year of their initial therapy (15 months vs. 6.5 months) [19].

\section{Second primary versus recurrent tumor}

As time to recurrence extended from 1st diagnosis, it is difficult to differentiate between delayed recurrence and second primary cancer. Second primary cancers might relatively respond well to 2 nd course of treatment than pure recurrent tumors in a previously irradiation bed due to inherent radioresistance of recurrent tumor and offensive nature of recurrent tumor cells. Several studies have reported data supporting this postulation [41]. RTOG 9610 study, have reported that the treatment outcome of each category of patients: 1-year OS rate and median survival for 2 nd primary cancer were $54 \%$ and 19.8 months, respectively, compared to $38 \%$ and 7.7 months, respectively, for recurrent cancers [19]. Stevens et al. [7] have reported on the outcomes of 100 patients treated with reirradiation alone. Their result showed that the 5 -year $0 S$ and LRC are better for 2 nd primary cancers compared to recurrent tumors (37\% and 60\%, for 2nd primary vs. $17 \%$ and $27 \%$, for recurrence in a previously irradiated field).

\section{Dose-response}

Several reports have reported that total administered radiation 
dose is a main predictive factor for outcome in re-irradiated tumors [22]. Theoretically, high dose radiation is needed because acquired radio-resistant cell population could be the source of recurrence. In general, lower radiation doses than that of original radiation treatment cannot be curative. Even though aggressive therapy with high-dose radiation and chemotherapy, locoregional sites are the major treatment of failure, indicating that main source of failure would be the remaining proportion of radioresistant cells in recurrent tumors [42]. One study has demonstrated the dose-response relationship that the median survival of 11.3 months and 2-year OS of 35\% for patients administered $\geq 58 \mathrm{~Gy}$, compared to 6.5 months and $8 \%$, for patients received lesser dose [43].

\section{Prognostic Factors Associated with Severe Grade 3 Toxicities}

Tumor recurrence is deemed to be the consequence of regrowth of radioresistant clonogens that survived through the initial treatment of RT. It is hard to control radioresistant population with repeated $R T$ in same scheme. From this reason, larger, intensive dose of $\mathrm{RT}$ are fundamentally required. However, in a reirradiation scenario, radiation oncologists have to make a judgment between the efficacy and morbidity caused by aggressive reirradiation. Optimal cutoff doses have been established based on the several dose-response relationship studies $[22,42,43]$. The cutting point dose expecting local control would be usually set at around $60 \mathrm{~Gy}$. Extreme caution is necessary if higher dose above $60 \mathrm{~Gy}$ is used. Salvage reirradiation with high curable dose have not been rationalized in price of excessive morbidity and deterioration of patient's QOL. Thus, before determining the reirradiation dose, radiation oncologist must cautiously consider on a lot of points such as target volume of reirradiation, proximity of critical dose limiting normal organs, the accuracy of high precision radiation delivery technique, and time interval from the first RT course. Risk factors associated with severe grade 3 toxicities are summarized in Table 4.

Among these factors, one must give a special attention to reirradiation volume and whether to administer concurrent chemotherapy.

Despite the increased use of high precision IMRT, toxicity results at the MD Anderson Cancer Center showed that the actuarial rate of any grade 3 toxicity approached 32\% at 2 years and $48 \%$ at 5 years. Predictors of grade 3 toxicity on multivariate analysis, patients with CTV larger than $50 \mathrm{~cm}^{3}$ were more likely to experience grade 3 toxicity $(H R, 3.11 ; p=$
0.003). Those who received concurrent chemotherapy were also more likely to experience grade 4 toxicity $(H R, 1.78 ; p=$ 0.035) [30]. Patients retreatment CTV of $25 \mathrm{~cm}^{3}(n=24)$ did not experience any grade 3 toxicity. They concluded that CCRT, reirradiation dose, and large radiation volume are predicting factors associated with high grade toxicities [30].

Especially, the mortality rate of CCRT can be substantially high up to 20\% [44]. The main causes of death in patients who have received prior chemotherapy are at increased risk of fatal toxicity such as neutropenic sepsis.

\section{Reirradiation in Combination with Novel Systemic Agent}

Whether the combination of systemic therapy to RT can increase the efficacy of reirradiation is currently unclear. No direct comparison studies of reirradiation alone vs. combined modality therapy have been undertaken. Large randomized studies comparing definite RT alone vs. chemotherapy-RT or meta-analysis in HNSCC have reported a survival benefit of about $6 \%$ in the cisplatin containing chemotherapyRT and concurrent cetuximab with definitive RT. In case of reirradiation setting, benefits of combined chemo-RT are likely to be similar to upfront therapy as in large randomized studies.

Choe et al. [44] reported an interesting result of 166 previously irradiated patients with non-metastatic HNSCC treated with CCRT. Their results were divided according to whether to administer chemotherapy before reirradiation. In a study by Choe et al. [44] an intriguing finding was reported; superior 2-year OS (28.4\% vs. $10.8 \% ; p=0.0043)$ and DFS ( $p=$ 0.008 ) rates in chemotherapy naive patients.

A comparable result has been demonstrated by Nagar et al. [45] that patients initially treated with RT alone gained higher DFS and OS ( $p=0.01$ for OS, $p=0.008$ for DFS) than those patients initially treated with CCRT [45]. One can hypothesize that previous full dose of CCRT are more likely to induce a more extensive fibrosis in the prior irradiated tissue and such a defective vascularity and fibrosis might compromise drug delivery. Also, RT-resistant hypoxic areas are certainly increased. Consequently, CCRT as a retreatment may not be as effective as upfront CCRT.

Several kinds of chemotherapeutic agents have been testified as concurrent regimen with reirradiation. Since the studies were small sized, performed in retrospective nature and the study design was heterogeneous, definitive assessment for efficacy and safety of tested regimens are unreliable.

More data is already available for cetuximab as effective 
regimen in reirradiation setting, when it is used in combination with RT in cetuximab-naive patients. Furthermore, its toxicity profile is significantly lower and acceptable compared to platinum-based or other chemotherapeutic regimens commonly utilized in patients of HNSCC. Cetuximab and reirradiation were used in two large studies. Heron et al. [28] have treated 35 matched patients with concurrent cetuximab and SABR $(5 \times 8$ Gy delivered every other day) vs. SABR alone in recurrent HNSCC. Cetuximab + SABR group showed higher local control rate than for the SABR alone group (49.2\% and 33.6\%; HR for local progression, $0.37 ; p=0.009$ ). Cetuximab + SABR group showed a clear difference in 2-year OS rates (53.3\% and $21.1 \%$, respectively; HR for death, $0.59 ; p=$ 0.031). This better survival of cetuximab-SABR group was observed in both patients group who received cetuximab during the upfront and re-treatment courses. There was no grade 4 acute toxicity. The incidence of late grade 3 toxicities in the cetuximab + SABR group and SABR-alone group were sufficiently low as 6\% and $3 \%$ respectively. In another report, Vargo et al. [46] have compared $\mathrm{QOL}$ of patients who were treated with concurrent cetuximab-SABR with SABR alone (40-50 Gy in 5 fractions). Concurrent administration of cetuximab to SABR had shown no additional influence on $\mathrm{OOL}$.

In summary, these two studies, defined that cetuximab is a promising systemic agent while maintaining $00 \mathrm{~L}$ with acceptable toxicities. Therefore, in routine clinical practice, cetuximab should be regarded as radiosensitizing concurrent regimen during reirradiation with tolerable toxicities profile.

\section{Summary and Patient Selection Algorithm for Reirradiation}

Most important step in patients with recurrent or 2 nd primary tumors is a thorough restaging evaluation prior to committing the patient to aggressive therapy with curative intent, regardless of surgery or reirradiation. Imaging studies to evaluate the extent of the disease include computed tomography and magnetic resonance, with further studies such as PET-CT, chest CT, or abdomen-pelvis CT performed to rule out metastatic diseases.

In addition to defining the extent of disease, essential step to planning the optimal treatment strategy should be assessment of the current patient's status including age performance status, accompanying comorbidities and life

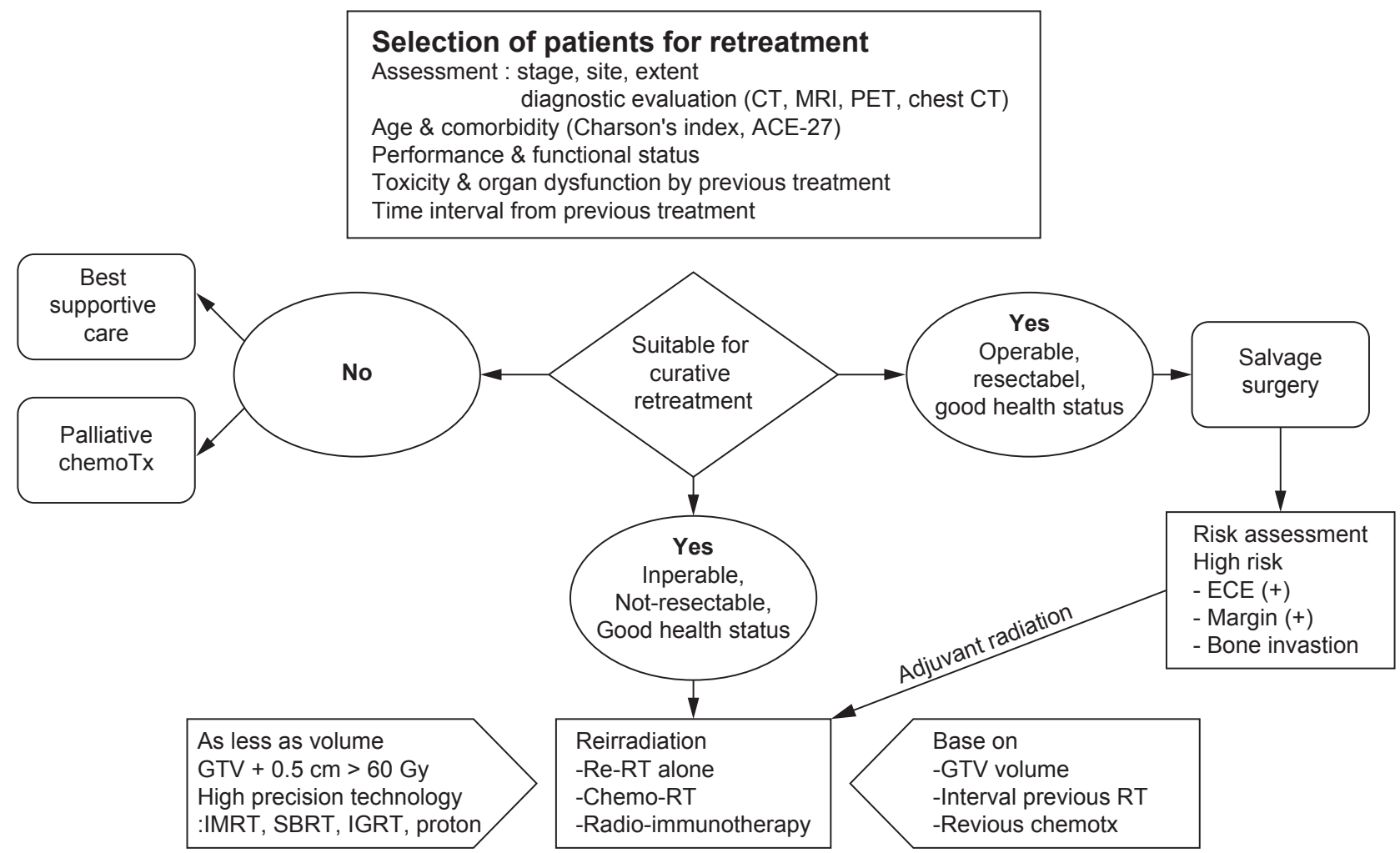

Fig. 1. Schematic presentation of patient selection and management guideline for reirradiation of recurrent or 2 nd primary head and neck cancer. 
expectancy, impairment of speech and swallowing function following prior therapy, nutritional status, severity of current symptoms, patient's willing to do retreatment and most importantly, the squeal of prior treatment such as fibrosis, carotid stenosis, dysphagia, xerostomia, and ORN. Patients with metastatic diseases and who are unsuitable for curative treatment with poor performance status, or who experienced severe toxicity from prior radiation should not be recommended for reirradiation.

Besides careful patient selection, all evaluation and treatment should be carried out at tertiary care centers with a multidisciplinary head and neck oncology team equipped with plenty of resources and experience to manage the complexities and toxicities of retreatment. A patient selection algorithm for reirradiation is presented in Fig. 1.

\section{Resectable recurrent diseases}

For operable and respectable recurrent disease, surgical resection is thought to be the standard of care. It may provide relatively long-term disease control. Unfortunately, although with complete surgical resection of recurrent disease, local failure rate reached up to 59\% [14]. Randomized data support the role of postoperative adjuvant reirradiation with or without chemotherapy to increase locoregional control and DFS for high-risk patients (gross residual disease, involved margins, or ECE) [15].

\section{Unresectable recurrent diseases}

A majority of patients with recurrent HNSCC are not committed to surgical resection because the disease is unsuitable for resection and patients are medically unfit for surgery. In these patients, palliative chemotherapy is considered as first available treatment. Although systemic chemotherapy might show a response rate about 35\%, response durations are relatively short and expectation of long-term survival is extremely scarce. Recent data of new biological agents may improve their outcomes in the future.

For some patients with unresectable disease but in good medical health status, reirradiation with or without chemotherapy (including biologic agents) is potentially a curative option. Patient selection for reirradiation is critical. For patients who are selected for curative intent reirradiation, high radiation dose $\geq 60 \mathrm{~Gy}$ is recommended. Smaller reirradiation volume without elective nodal area appeared to be reasonable. Conventional fractionation or hyperfractionation without acceleration with a minimum 6-hour interval is favored. High precision IMRT technique must be applied with high priority to the limited target volume to reduce acute and late morbidities and to improve local control.

New conformal radiation therapy including SABR, hypofractionated stereotactic radiotherapy, and proton therapy may be particularly selected in some cases. Additional data are required to define which patient subgroups are most likely to benefit from these new modalities [47].

\section{Conflict of Interest}

No potential conflict of interest relevant to this article was reported.

\section{References}

1. Cooper JS, Zhang 0 , Pajak TF, et al. Long-term follow-up of the RTOG 9501/intergroup phase III trial: postoperative concurrent radiation therapy and chemotherapy in high-risk squamous cell carcinoma of the head and neck. Int J Radiat Oncol Biol Phys 2012;84:1198-205.

2. Brockstein B, Haraf DJ, Rademaker AW, et al. Patterns of failure, prognostic factors and survival in locoregionally advanced head and neck cancer treated with concomitant chemoradiotherapy: a 9-year, 337-patient, multi-institutional experience. Ann Oncol 2004;15:1179-86.

3. Cooper JS, Pajak TF, Rubin P, et al. Second malignancies in patients who have head and neck cancer: incidence, effect on survival and implications based on the RTOG experience. Int J Radiat Oncol Biol Phys 1989;17:449-56.

4. Bachar GY, Goh C, Goldstein DP, O'Sullivan B, Irish JC. Longterm outcome analysis after surgical salvage for recurrent tonsil carcinoma following radical radiotherapy. Eur Arch Otorhinolaryngol 2010;267:295-301.

5. Vermorken JB, Mesia R, Rivera $F$, et al. Platinum-based chemotherapy plus cetuximab in head and neck cancer. $\mathrm{N}$ Engl J Med 2008;359:1116-27.

6. Weichselbaum RR, Beckett MA, Schwartz $J$, Dritschilo A. Radioresistant tumor cells are present in head and neck carcinomas that recur after radiotherapy. Int J Radiat Oncol Biol Phys 1988;15:575-9.

7. Stevens KR Jr, Britsch A, Moss WT. High-dose reirradiation of head and neck cancer with curative intent. Int J Radiat Onco Biol Phys 1994;29:687-98.

8. Zbaren P, Nuyens M, Curschmann J, Stauffer E. Histologic characteristics and tumor spread of recurrent glottic carcinoma: analysis on whole-organ sections and comparison with tumor spread of primary glottic carcinomas. Head Neck 
2007;29:26-32.

9. McDonald MW, Moore MG, Johnstone PA. Risk of carotid blowout after reirradiation of the head and neck: a systematic review. Int J Radiat Oncol Biol Phys 2012;82:1083-9.

10. Ang KK, Jiang GL, Feng Y, Stephens LC, Tucker SL, Price RE. Extent and kinetics of recovery of occult spinal cord injury. Int J Radiat Oncol Biol Phys 2001;50:1013-20.

11. Kirkpatrick JP, van der Kogel AJ, Schultheiss TE. Radiation dose-volume effects in the spinal cord. Int J Radiat Oncol Biol Phys 2010;76(3 Suppl):S42-9.

12. Nieder C, Grosu AL, Andratschke NH, Molls M. Update of human spinal cord reirradiation tolerance based on additional data from 38 patients. Int J Radiat Oncol Biol Phys 2006;66:1446-9.

13. Goodwin WJ Jr. Salvage surgery for patients with recurrent squamous cell carcinoma of the upper aerodigestive tract: when do the ends justify the means? Laryngoscope 2000;110(3 Pt 2 Suppl 93):1-18.

14. Kim AJ, Suh JD, Sercarz JA, et al. Salvage surgery with free flap reconstruction: factors affecting outcome after treatment of recurrent head and neck squamous carcinoma. Laryngoscope 2007;117:1019-23.

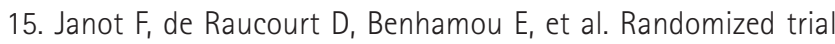
of postoperative reirradiation combined with chemotherapy after salvage surgery compared with salvage surgery alone in head and neck carcinoma. J Clin Oncol 2008;26:5518-23.

16. Strojan P, Corry J, Eisbruch $A$, et al. Recurrent and second primary squamous cell carcinoma of the head and neck: when and how to reirradiate. Head Neck 2015;37:134-50.

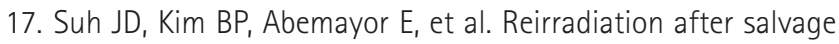
surgery and microvascular free flap reconstruction for recurrent head and neck carcinoma. Otolaryngol Head Neck Surg 2008;139:781-6.

18. Tortochaux J, Tao Y, Tournay E, et al. Randomized phase III trial (GORTEC 98-03) comparing re-irradiation plus chemotherapy versus methotrexate in patients with recurrent or a second primary head and neck squamous cell carcinoma, treated with a palliative intent. Radiother Oncol 2011;100:70-5.

19. Spencer SA, Harris J, Wheeler RH, et al. Final report of RTOG 9610, a multi-institutional trial of reirradiation and chemotherapy for unresectable recurrent squamous cell carcinoma of the head and neck. Head Neck 2008;30:281-8.

20. Langer CJ, Harris J, Horwitz EM, et al. Phase II study of lowdose paclitaxel and cisplatin in combination with splitcourse concomitant twice-daily reirradiation in recurrent squamous cell carcinoma of the head and neck: results of Radiation Therapy Oncology Group Protocol 9911. J Clin Oncol
2007;25:4800-5.

21. Argiris A, Li Y, Forastiere A. Prognostic factors and longterm survivorship in patients with recurrent or metastatic carcinoma of the head and neck. Cancer 2004;101:2222-9.

22. Lee $N$, Chan $K$, Bekelman JE, et al. Salvage re-irradiation for recurrent head and neck cancer. Int J Radiat Oncol Biol Phys 2007;68:731-40.

23. Kharofa J, Choong N, Wang D, et al. Continuous-course reirradiation with concurrent carboplatin and paclitaxel for locally recurrent, nonmetastatic squamous cell carcinoma of the head-and-neck. Int J Radiat Oncol Biol Phys 2012;83:690-5.

24. Siddiqui $F$, Patel $M$, Khan $M$, et al. Stereotactic body radiation therapy for primary, recurrent, and metastatic tumors in the head-and-neck region. Int J Radiat Oncol Biol Phys 2009;74:1047-53.

25. Heron $D E$, Ferris $R L$, Karamouzis $M$, et al. Stereotactic body radiotherapy for recurrent squamous cell carcinoma of the head and neck: results of a phase I dose-escalation trial. Int J Radiat Oncol Biol Phys 2009;75:1493-500.

26. Rwigema JC, Heron DE, Ferris $R L$, et al. Fractionated stereotactic body radiation therapy in the treatment of previously-irradiated recurrent head and neck carcinoma: updated report of the University of Pittsburgh experience. Am J Clin Oncol 2010;33:286-93.

27. Cengiz M, Ozyigit $G$, Yazici $G$, et al. Salvage reirradiaton with stereotactic body radiotherapy for locally recurrent head-andneck tumors. Int J Radiat Oncol Biol Phys 2011;81:104-9.

28. Heron DE, Rwigema JC, Gibson MK, Burton SA, Quinn AE, Ferris RL. Concurrent cetuximab with stereotactic body radiotherapy for recurrent squamous cell carcinoma of the head and neck: a single institution matched case-control study. Am J Clin Oncol 2011;34:165-72.

29. Lin R, Slater JD, Yonemoto LT, et al. Nasopharyngeal carcinoma: repeat treatment with conformal proton therapy: dose-volume histogram analysis. Radiology 1999;213:489-94.

30. Takiar V, Garden AS, Ma D, et al. Reirradiation of head and neck cancers with intensity modulated radiation therapy: outcomes and analyses. Int J Radiat Oncol Biol Phys 2016;95:1117-31.

31. Kuhn E, Molnar Z, Bohm K. Postirradiation changes on the lymphatics studied by lymphography. Rofo 1979;131:92-6.

32. Hoebers $F$, Heemsbergen $W$, Moor $S$, et al. Reirradiation for head-and-neck cancer: delicate balance between effectiveness and toxicity. Int J Radiat Oncol Biol Phys 2011;81:e111-8.

33. Sulman EP, Schwartz DL, Le $T$, et al. IMRT reirradiation of head and neck cancer-disease control and morbidity outcomes. Int J Radiat Oncol Biol Phys 2009;73:399-409. 
34. Yu KH, Leung SF, Tung SY, et al. Survival outcome of patients with nasopharyngeal carcinoma with first local failure: a study by the Hong Kong Nasopharyngeal Carcinoma Study Group. Head Neck 2005;27:397-405.

35. Patel PR, Salama JK. Reirradiation for recurrent head and neck cancer. Expert Rev Anticancer Ther 2012;12:1177-89.

36. Duprez F, Madani I, Bonte K, et al. Intensity-modulated radiotherapy for recurrent and second primary head and neck cancer in previously irradiated territory. Radiother Oncol 2009;93:563-9.

37. Langendijk JA, Kasperts $N$, Leemans $C R$, Doornaert $P$, Slotman BJ. A phase II study of primary reirradiation in squamous cell carcinoma of head and neck. Radiother Oncol 2006;78:30612.

38. De Crevoisier R, Bourhis J, Domenge $C$, et al. Full-dose reirradiation for unresectable head and neck carcinoma: experience at the Gustave-Roussy Institute in a series of 169 patients. J Clin Oncol 1998;16:3556-62.

39. Yamazaki H, Kodani N, Ogita M, Sato K, Himei K. Reirradiation of head and neck cancer focusing on hypofractionated stereotactic body radiation therapy. Radiat Oncol 2011;6:98.

40. Ohizumi Y, Tamai Y, Imamiya S, Akiba T. Prognostic factors of reirradiation for recurrent head and neck cancer. Am J Clin Oncol 2002;25:408-13.

41. Mendenhall WM, Mendenhall CM, Malyapa RS, Palta JR, Mendenhall NP. Re-irradiation of head and neck carcinoma.
Optimal reirradiation strategy for head and neck cancer

Am J Clin Oncol 2008;31:393-8.

42. Popovtzer A, Gluck I, Chepeha DB, et al. The pattern of failure after reirradiation of recurrent squamous cell head and neck cancer: implications for defining the targets. Int J Radiat Oncol Biol Phys 2009;74:1342-7.

43. Salama JK, Vokes EE, Chmura SJ, et al. Long-term outcome of concurrent chemotherapy and reirradiation for recurrent and second primary head-and-neck squamous cell carcinoma. Int J Radiat Oncol Biol Phys 2006;64:382-91.

44. Choe KS, Haraf DJ, Solanki A, et al. Prior chemoradiotherapy adversely impacts outcomes of recurrent and second primary head and neck cancer treated with concurrent chemotherapy and reirradiation. Cancer 2011;117:4671-8.

45. Nagar YS, Singh S, Datta NR. Chemo-reirradiation in persistent/recurrent head and neck cancers. Jpn J Clin Oncol 2004;34:61-8.

46. Vargo $J A$, Heron $D E$, Ferris $R L$, et al. Prospective evaluation of patient-reported quality-of-life outcomes following SBRT \pm cetuximab for locally-recurrent, previously-irradiated head and neck cancer. Radiother Oncol 2012;104:91-5.

47. McDonald MW, Lawson J, Garg MK, et al. ACR appropriateness criteria retreatment of recurrent head and neck cancer after prior definitive radiation expert panel on radiation oncology-head and neck cancer. Int J Radiat Oncol Biol Phys 2011;80:1292-8. 